\title{
Tailored Exercise with an Innovative Mechanical Device: Effects on Cervical-Dorsal Rachis
}

\author{
Luca Valerio Messa ${ }^{1, *}$, Alessandro Biffi ${ }^{2}$, Fredrick Fernando ${ }^{2}$, Federica Ginanneschi ${ }^{1}$ and \\ Alessandro Rossi ${ }^{1}$ \\ 1 Department of Medical, Surgery and Neurological Sciences, University of Siena, 53100 Siena, Italy; \\ ginanneschi@unisi.it (F.G.); rossiale@unisi.it (A.R.) \\ 2 Med-Ex, Medical Partner Scuderia Ferrari, 00187 Roma, Italy; alessandro.biffi@med-ex.it (A.B.); \\ fred@med-ex.it (F.F.) \\ * Correspondence: lucavalerio.messa@gmail.com; Tel.: +39-339-343-5001
}

Academic Editor: Giuseppe Musumeci

Received: 6 January 2016; Accepted: 11 April 2016; Published: 15 April 2016

\begin{abstract}
Trapezius muscle has a fundamental role in cervico-dorsal pain and posture. Often, in movements involving trapezius, the predominant activity is carried out by the upper trapezius, and many times this may be a risk factor for the integrity of the cervico-dorsal structures. To investigate the effects of physical exercise with the new device called the "Angel's Wings" on a sample of different professionals. We enrolled 15 volunteers for electromyography (EMG) data, which was collected during the physical performance; 3 volunteers for magnetic resonance imaging (MRI) data, collected before and after a training period; and 73 workers of Ferrari S.p.A. and the Scuderia Ferrari racing team division for a visual analogue scale (VAS) pain score, collected before and after one session of training. EMG shows a decoupling of upper and lower trapezius activity; MRI shows a realignment of cervical-dorsal rachis after one month of training; VAS pain score significantly decreased after the physical exercise with the "Angel's Wings". Results show that the use of the "Angel's Wings" is applicable to counteract and decrease the neck pain by a decontraction of the upper trapezius and correct postural defects of the cervico-dorsal column.
\end{abstract}

Keywords: Angel's Wings; neck pain; occupational medicine

\section{Introduction}

There is solid evidence that physical exercise, physical training, fitness and wellness programs can sharply decrease absence at work, improving the quality of occupational performance, regardless of the type of job. In fact, in addition to the well-known health benefits of being physically fit, physical training interventions can prevent limitations in job performance due to inadequate muscle effectiveness as well as possible mismatches between job demands and physical capacities. In designing a physical training program for improving occupational performance, several fundamental program-design variables should be considered: exercise selection and order; equipment used; specificity; frequency; sets; repetitions; rest intervals; duration; variation; and progression [1-3]. Because the physical training should be done during the workday, it should be fast, effective, and efficient; moreover, it would be useful if every company had at least a small gymnasium.

Neck and shoulder disorders are a considerable health problem in the working population with prevalence rates of equal or higher than 30\% [4]. Neck pain is common among adults in developed countries and contributes importantly to the demand for medical services and the economic burden of absence from work due to sickness $[5,6]$. Although the etiology of work-related neck disorders is likely multidimensional, strong evidence has been found for a causal relationship between neck disorders and highly repetitive work, forceful exertions, a high level of static contractions, prolonged static loads and extreme postures, and combinations of these factors. 
Altered trapezius activity has been linked to neck pain, and previous studies have shown that individuals with neck pain display altered trapezius activation patterns $[7,8]$. In particular, the deleterious effect of sustained activity pattern in the upper trapezius (UT) muscle (which is the occurrence of continuous muscle activation without interruptions) has been proposed as a common mechanism for explaining neck and shoulder pain $[4,7,8]$.

Because the physical exercises that are proposed for exercising the lower trapezius (LT) muscle always make the UT work more than LT [9], there is a need of a device that would involve synergistically the cervical-dorsal muscles. In particular, there is a need to emphasize the activity of the LT, while concurrently relaxing the UT to reduce the consequences of faulty posture and of excessive activation of the cervical-dorsal muscles. This physical exercise should be able to decouple and then fractionate the UT and the LT muscle activity.

In order to test this proposal, we used a specially designed device called the "Angel's Wings" (patent number 0001401430). The device acts mainly on the axial muscles of the cervical-dorsal rachis, and thanks to a simple distribution of vectors of forces, it is possible to isolate the LT activity from that of the UT, so it can correct posture of this tract [10]. In the present paper, we tested, for the first time, the ability of the Angel's Wings to fractionate the UT and the LT muscle activity, and the impact of decoupling this muscle on posture over a period of 30 days. In a second stage, we tested the Angel's Wings, and the method that we propose on a sample of 73 employees of Ferrari S.p.A. and the Scuderia Ferrari racing team division, based in Maranello, Italy, to determine whether there was neck and shoulder pain relief following one session of the proposed exercise.

\section{Materials and Methods}

\subsection{Subjects}

Fifteen healthy volunteers ( 9 males and 6 females, aged between 22 and 67 years $(44.5 \pm 31.8)$ ) performed surface electromyographic (sEMG) analysis, and 3 healthy volunteers ( 3 males, aged between 56 and 67 years $(61.5 \pm 7.7)$ ) were evaluated with magnetic resonance imaging (MRI). Subsequently, for the visual analogue scale (VAS) evaluation, 73 workers were enrolled (68 males, 5 females; aged between 18 and 70 years $(44 \pm 36.7)$ ): 5 pilots; 10 car mechanics and pit stop laborers; 25 warehousemen, and 33 engineers. Most of the subjects suffered, although of varying severity, from neck and shoulder pain that sometimes radiated to the dorsal portion. All workers had no history or clinical signs suggesting systemic disease apart from pain. Each of the participants compiled an informed consent.

\subsection{Device}

The aim of the Angel's Wings device (Figure 1A) is the distension of the cervical-dorsal spine, with the contemporary rehabilitation of the shoulder joint position in its natural seat, rather than rotated forward. The Angel's Wings is a device consisting of a seat and a system of pulleys and cables connected to the adjustable weights that can be lifted by the user with two handles. In the positive phase of the movement, the weight, attached to the cables, is lifted perpendicularly, and, during the return phase, it is brought back to its original position. The weight always moves along a straight line perpendicular to the ground and on the same sagittal plane of the spine via a system of pulleys. What makes the relaxation of the UT possible is the fact that the two lateral pulleys of the machine allow the user to obtain a starting position with the external section of cables parallel to the ground.

Since distension of the forearms takes place in the frontal plane and the tension of the cable, in the section parallel to the ground, is directly proportional to the tension of the cable in the stretch perpendicular, and therefore to the force required to lift the weight, such force is discharged from the UT to the LT. The seat is adjusted taking into account the length of the arms of the user so that, once the handles are grasped, the cables are parallel to the ground. The starting position is then seated with the grab handles and palms facing the direction of the eyes. Trying to keep the shoulders as 
straight as possible, the spinal column (including the neck) outstretched, and the back flat and in contact with the backrest, the forearms are extended outward. It is important to try to keep the elbows as still as possible, as if they were stuck in a fixed point. The return to the starting position must be slow; movements should never be carried out too quickly. The execution of the task is outlined in Figure 1B-E: Starting from a seated position (Figure 1B: front; Figure 1D: lateral) the user extends the forearms (Figure 1C: front; Figure 1E: lateral), keeping elbows at shoulder height, to lift a weight through the cables of the equipment. The forearms move in the frontal plane. In this device and method of use, no more than $7 \mathrm{~kg}$ are lifted. The motor task described consists in the lifting of a weight with specific equipment used in definite positions. Therefore, to obtain the best conditions, there were experts present to teach the sample of subjects how to do the exercise in the best way, and to correct the users when they were doing the movement wrong or not completely correct. Moreover, the users had a mirror in front of them to obtain real-time feedback on their movements.

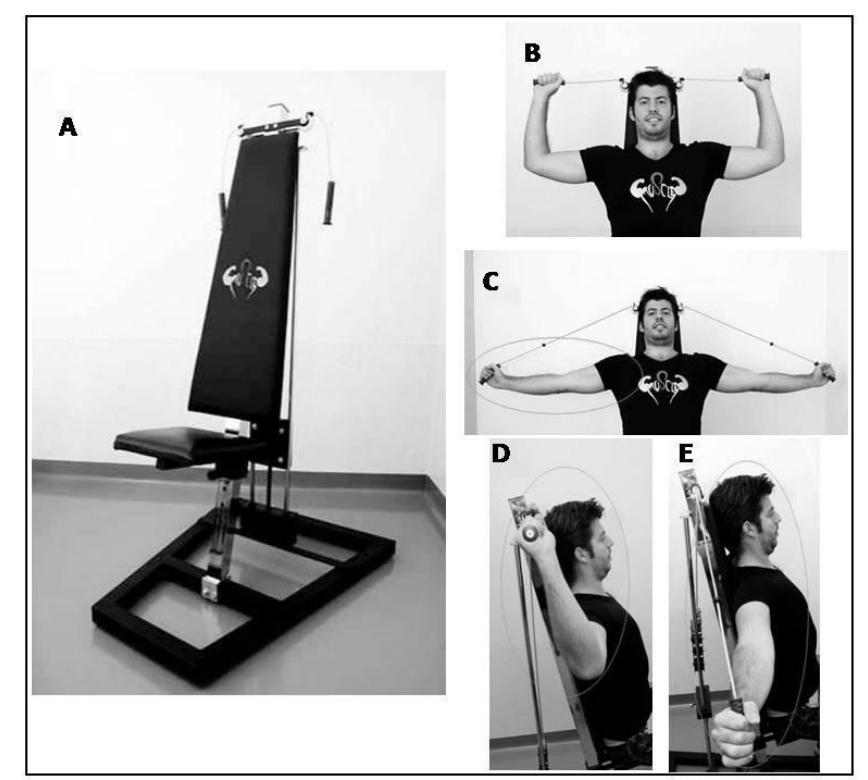

Figure 1. The Angel's Wings device (A); front (B) and lateral (D) view of the starting position of the exercise; front $(\mathbf{C})$ and lateral (E) view of the distension of the forearms.

\subsection{Electromyographic and Imaging Studies}

The EMG studies were conducted in different sessions with 2 different equipment: electromyography Medelec Sapphyre 1P and EMG TruTrace. Disposable surface AG/ AgCl electrodes (auto-adhesive hydrogel, $15 \mathrm{~mm} \times 20 \mathrm{~mm}$ ) were used for each subject. A bipolar electrode montage was used. The active electrodes were located at $50 \%$ on the line from the acromion to the spine on vertebra C7 (for the UT) and in a line connecting T8 with the trigonum spinea (for the LT). The inter-electrode distance was $2 \mathrm{~cm}$. The electrodes location was directed parallel to the muscle fiber. The ground electrode was located on clavicle. The skin under the recording electrodes was carefully prepared with an abrasive past. Electromyography was performed in order to verify the activity of UT and LT during the device-drive activity. The obtained EMG signals were filtered $(20-500 \mathrm{~Hz})$ and stored for the analysis. The raw EMG signal was translated to a single polarity to facilitate signal processing, and a full-wave rectification was made.

Among each subject's trial, the four values were normalized to the maximum (expressed as a percentage). This analysis was made severally for the UT and LT. Magnetic resonance imaging (MRI) was conducted on the cervico-dorsal rachis. All MRIs (Avanto 1,5 T; A.G. SIEMENS, Erlangen, Germany) were reviewed by one expert neuroradiologist, blinded to the study protocol. The MRI was performed to investigate the effects induced by physical exercise performed with the Angel's 
Wings over a training period of 30 days. The measurements to explore the morphological changes were performed with the software built into the MRI system (Syngo 8.4 version).

\subsection{Study Protocol}

\subsubsection{Preliminary Studies}

In the preliminary study, sEMG of UT and LT muscles were performed in 9 healthy volunteers during the 4 phases of the Motor Task, or rather in the dynamic execution of the exercise: (1) start position, i.e., when the user is ready to start the exercise like in Figure 1B; (2) contraction during lifting, i.e., all the movement in which the forearms extend outwardly moving toward the position depicted in Figure 1C; (3) return phase, i.e., when the forearms are returning to the position of Phase 1; and (4) final phase of rest, which corresponds exactly to the start position, and the user is ready to repeat the exercise. Surface EMG was also performed in 6 subjects with a different device, but no differences in the signal of the performance between the two EMG procedures used were found. Data were tested for normal distribution (the Kolmogorov-Smirnov distance method) and a parametric test was used. One-way analysis of variance (one-way ANOVA) and Tukey's test for multiple comparison was used to analyze the variations in the muscle activity in the UT and LT. Then, 3 healthy volunteers were examined via MRI at the beginning and at the end of a training period with the Angel's Wings. The proposed protocol for the training, during the period of this preliminary study, was of $2+2 \mathrm{~min}$ of performance duration (lifting a weight of $4 \mathrm{~kg}$ ), with a recovery interval of $2 \mathrm{~min}$. The 3 subjects who underwent MRI performed the motor task daily (morning and evening) for 30 days. There were 2 MRI measurements performed on the scans of the supine subject. The first measurement was of the angle obtained from the intersection of the line drawn between the intervertebral spaces D4/D5 and $\mathrm{C} 6 / \mathrm{C} 7$ and the line perpendicular to the horizontal plane; the angle was the index of curvature of the cervico-dorsal junction. The second was of the angle obtained from the intersection of the line drawn between the intervertebral space $\mathrm{C} 6 / \mathrm{C} 7$ and the opisthion and the line perpendicular to the horizontal plane; the angle was the index of the decrease in the cervical curvature.

\subsubsection{Occupational Study}

We tested the device and the respective physical exercise on a sample of 73 employees. Each of the participants compiled the first part of a questionnaire before the protocol of exercise in which they had to report their personal sensation in general of the pain for the indicated portions of body: neck, shoulders, and the dorsal muscles. The pain was quantified, before and after the protocol, by the standardized VAS score for pain (0-10 interval). The proposed method for the 73 employees consisted in three series of physical exercise carried out with the Angel's Wings, with each series 2 min long, with a recovery interval of 2 min between the series for every session made. For each participant, the first series was performed with a weight of $3 \mathrm{~kg}$, the second with $5 \mathrm{~kg}$, and the last one with $4 \mathrm{~kg}$. Each of the participants, after being trained, completed one session with the proposed device. After the session, each of the participants were asked to compile the last part of the questionnaire in which the same VAS score for pain was used to quantify the same portions of the body. Data were tested for normal distribution (the Kolmogorov-Smirnov distance method) and a non-parametric test was used. The statistical differences were calculated using the Wilcoxon matched-pairs signed rank test. Moreover, each of the participants was asked to answer a final anonymous questionnaire in which they had to report their impressions of the effect produced by the use of this device, choosing between Not Good, Little Good, Good, Very Good, Optimal, and their impressions about how they felt after the session, choosing between No Effect, Little Better, Better, Much Better. 


\section{Results}

\subsection{Preliminary Studies}

The graph in Figure 2 shows the percentage variation of the mean of UT activity and of the mean of LT activity, during the four phases $(n=15)$. Table 1 summarizes the mean of the percentage variations in maximum amplitudes of EMG on UT and LT, during the four phases of the task, considering Phase 1 (start position) equal to 0 for both portions of the trapezius muscle and the relative statistically differences. A statistically significant difference in Phase 2 (contraction during lifting) was found (see Table 1 for statistical results).

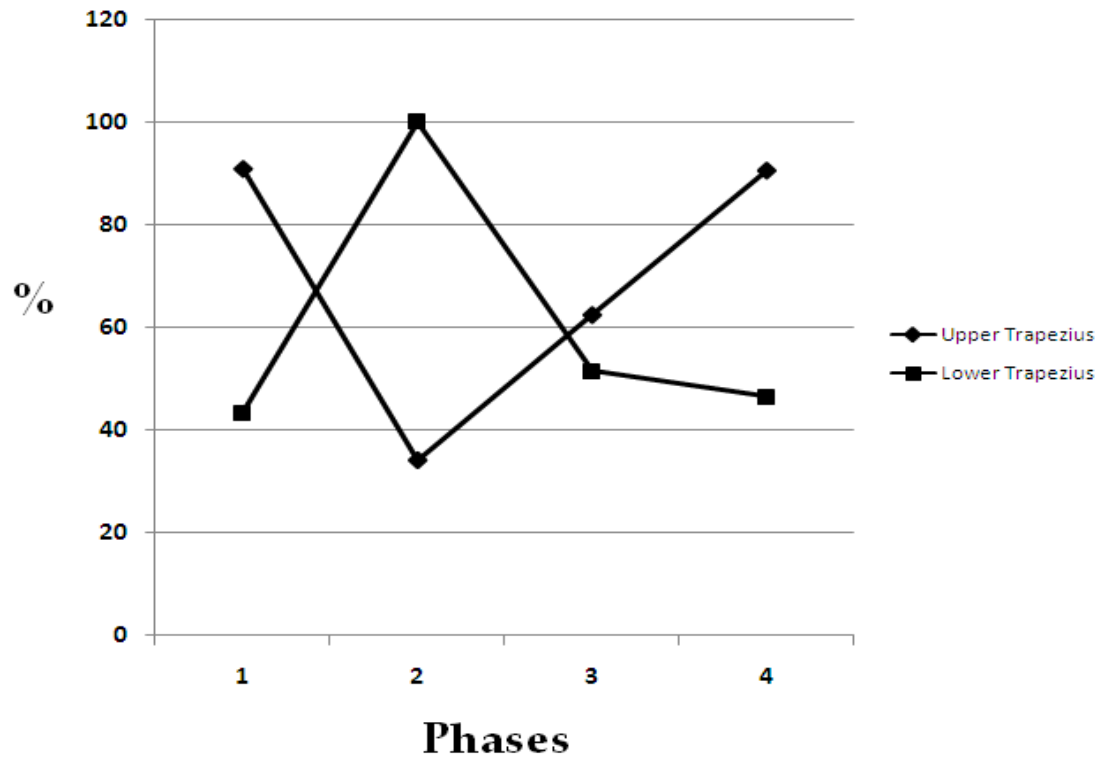

Figure 2. Mean amplitude of upper trapezius activity and lower trapezius activity during the four phases of the exercise. For every single subject's trial, the maximum value in $\mathrm{mV}$ among the phases was considered as $100 \%$, and all the other values in $\mathrm{mV}$ of every trial changed to a percentage of it for every subject $(n=15)$.

Table 1. Percentage changes of electromyography activity in upper trapezius and lower trapezius, during the four phases of the task, with the statistical analysis. We used a one-way ANOVA and Tukey's test for multiple comparison. A statistically significant difference in Phase 2 (contraction during lifting) was found.

\begin{tabular}{cccc}
\hline Phase & Right Upper Trapezius & Right Lower Trapezius & Tukey's Test \\
\hline 1 & $90.88 \pm 10.82$ & $43.57 \pm 13.95$ & $p>0.05$ \\
2 & $34.2 \pm 12.82$ & $100 \pm 0$ & $p<0.01$ \\
3 & $62.45 \pm 12.81$ & $51.66 \pm 15.9$ & $p>0.05$ \\
4 & $90.57 \pm 12.5$ & $46.64 \pm 11.71$ & $p>0.05$ \\
\hline
\end{tabular}

Figure 3 shows the MRI results of one of the three subjects (64 years) who was trained for 30 days with the Angel's Wings. The figure shows the post-training variations in the curvatures of the cervico-dorsal (see Figure 3B) and cervical (see Figure 3D) column compared to those of the pre-training (see Figure 3A,C). 

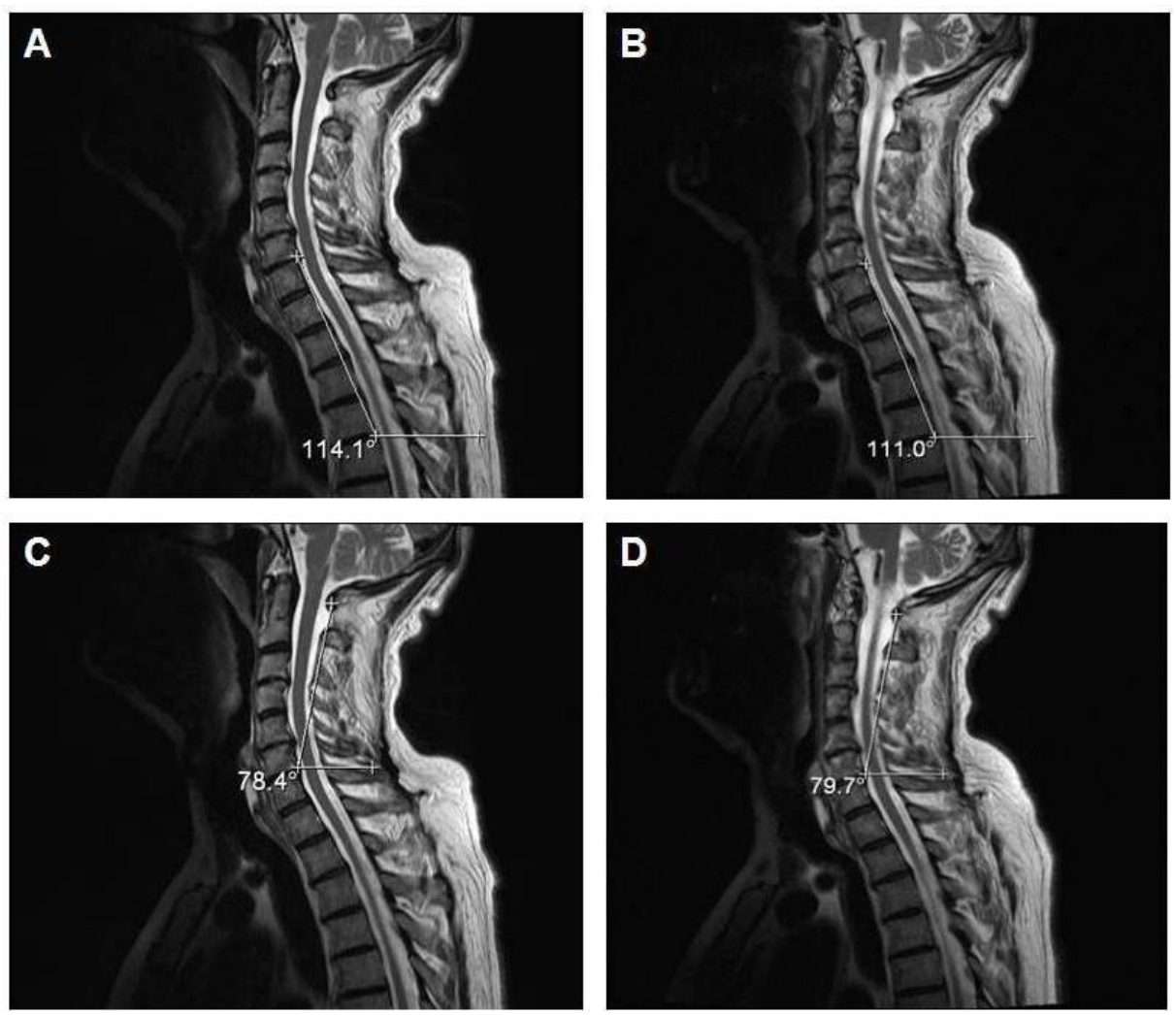

Figure 3. Pre-exercise (A and $\mathbf{C}$ ) and post-exercise (B and D) magnetic resonance imaging (MRI) images (sagittal T2 weighted fast spin echo images; TR/TE-4000/105) of one representative subject who performed the motor task daily (morning and evening) for a duration of 30 days.

\subsection{Occupational Study}

The graph in Figure 4 shows the averages of the reported pain $(n=73)$ before and after the treatment with the Angel's Wings for three parts of the body (i.e., neck, shoulders, and dorsal section).

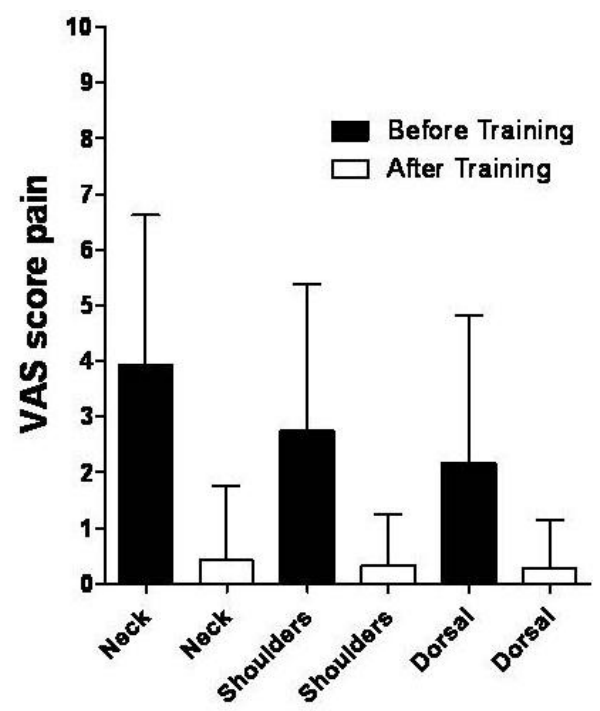

Figure 4. Mean visual analogue scale (VAS) score and the respective SD obtained before and after the training with the Angel's Wings. The data refer to all subjects $(n=73)$. For all body portions considered, a statistical significant difference between before and after training was found $(p<0.0001)$. 
For the neck and shoulders, a mean was calculated considering exclusively the subjects reporting a painful symptomatology of the respective body portion. In the whole group of 73 subjects, 60 subjects reported pain in the neck, and 49 subjects reported pain in the shoulders. The graph in Figure 5 shows the mean $(n=60)$ and the respective statistical differences of the VAS score with respect to neck pain (Figure 5A) and shoulder pain (Figure 5B) before and after the training with the Angel's Wings.
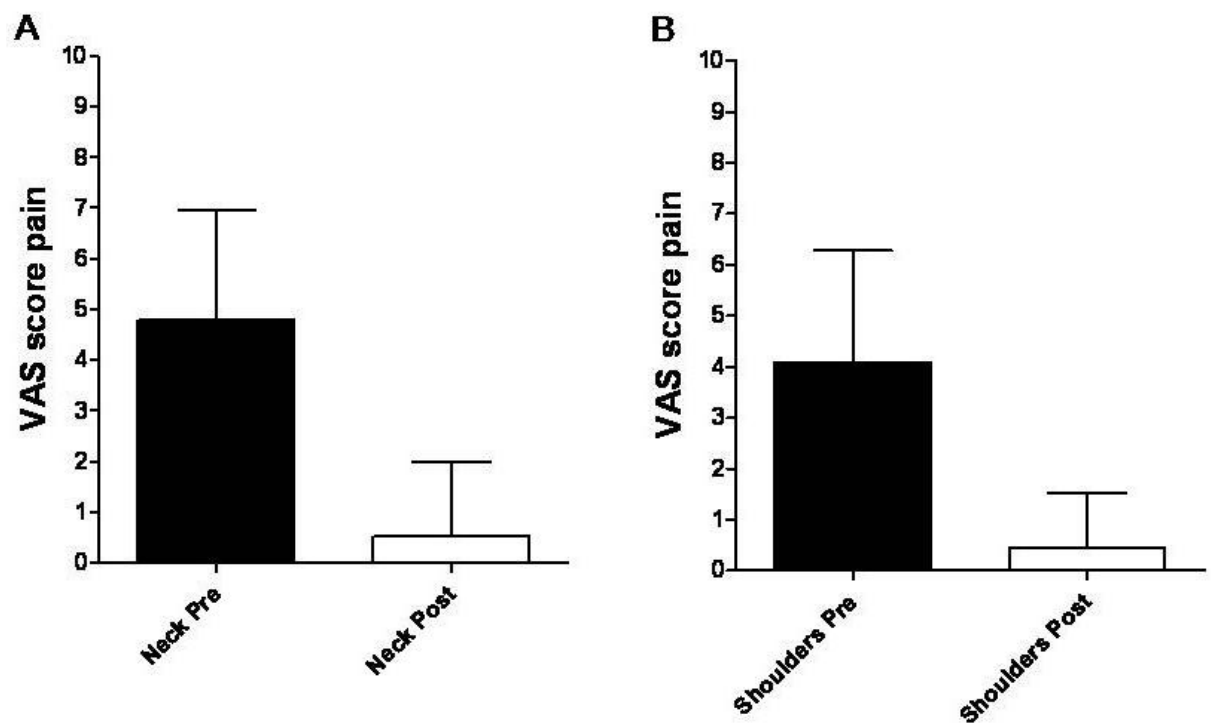

Figure 5. Mean VAS score and the respective SD obtained before and after the training with the Angel's Wings. The data refer to subjects $(n=60)$ reporting a painful symptomatology of the neck $(\mathbf{A})$ and to subjects $(n=49)$ reporting a painful symptomatology of the shoulders $(\mathbf{B})$. For both graphs, a statistical significant difference between before and after training was found $(p<0.0001)$.

Finally, Figure 6 shows the percentages of the validity assessment by the tested population regarding the physical exercise with the Angel's Wings (A) and the percentages of the improvement assessment, always reported by the tested population, regarding the sensation after the use of the Angel's Wings (B).

\section{A Percentages Validity assessment}

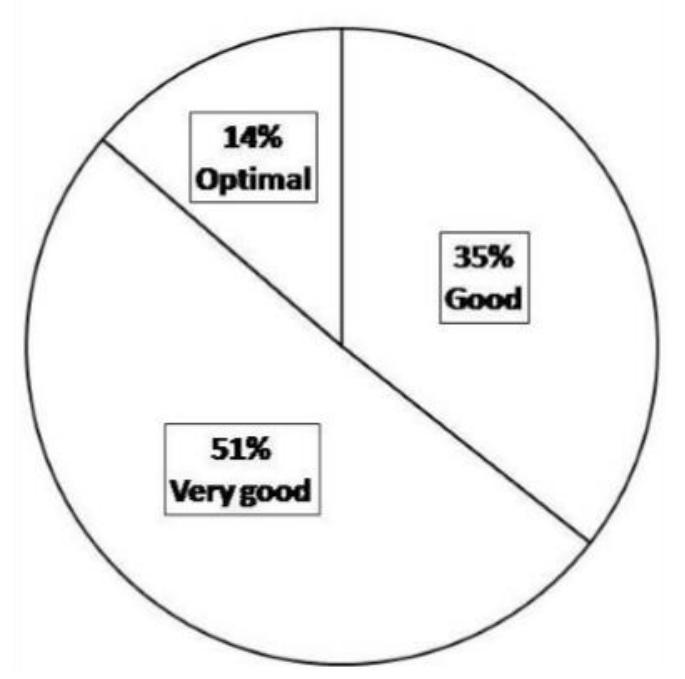

\section{B Percentages Improvement assessment}

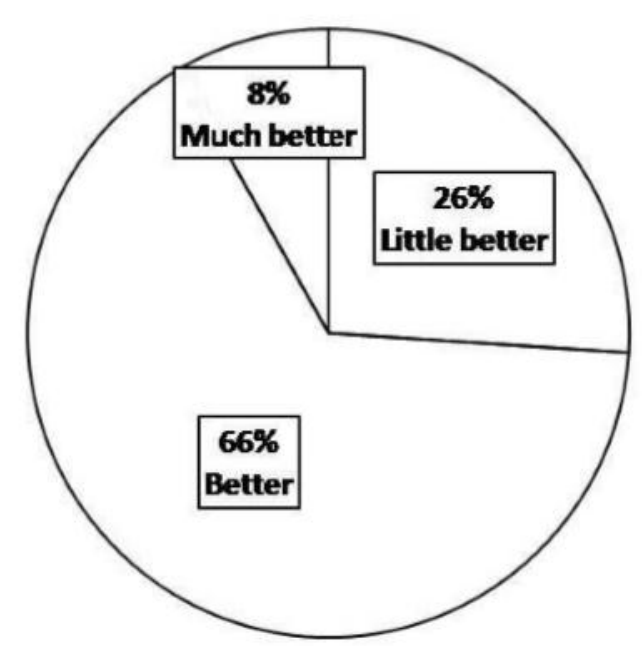

Figure 6. Percentages of the validity assessment (A) and of the improvement assessment (B). 


\section{Discussion}

The trapezius muscle is a very large muscle area that extends longitudinally from the occipital bone to the lower thoracic vertebrae and laterally to the spine of the scapula. The trapezius supports the shoulder as well as the neck. Being the primary actors in an upward scapular rotation, UT and LT should make adequate and relatively equal contributions to maintain normal biomechanics. Most commonly, the UT is believed to influence pain in the neck. In effect, individuals with neck and shoulder pain display altered trapezius activation patterns, with UT muscle more active than the LT [4] and limited strength or endurance of LT muscle [11,12]. The over-activity of UT will results in scapulothoracic muscle imbalance causing weakness of LT muscle with possible irradiation of the pain downward. For these reasons, it would be appropriate to emphasize LT strengthening with exercises that simultaneously induce a relaxation of the UT.

The Angel's Wings represent the first device able to decouple the UT and the LT muscle activity, emphasizing the muscular activity of the LT and then the relaxation of the UT. This characteristic indeed distinguishes Angel's Wings from other physical exercises that act simultaneously on all trapezius segments. The exercise with Angel's Wings is absolutely not difficult and produces significant pain relief in the body portions on which it acts, namely, the neck, the shoulders, and the back. Since it is a physical exercise designed to make the obtained benefits stable and long-lasting, a hypothetical user should train with this device and protocol with constancy-for example, at least three times per week, as suggested by previous guidelines regarding physical training [13]. However, an initial feeling of great relaxation and of pain decrease can be felt after only one session. Moreover, the fact that, regardless of gender, age, or level of training, the weight lifted was within a range between 1 and $7 \mathrm{~kg}$ indicates that the stimulated muscles in this performance by the proposed device have almost the same degree of training in all persons, corroborating the idea that this physical exercise is most likely suitable for everyone.

The most important phase of the motor task with the Angel's Wings is the Phase 2, i.e., the contraction during lifting. The sEMG clearly show that, during Phase 2, a sharp contraction of the LT corresponds to a large relaxation of the UT. Since altered trapezius activity has been linked to neck pain and previous studies have shown that individuals with neck pain display altered trapezius activation patterns [7,8], we hypothesize that this particular trapezius activity, obtained with the use of the Angel's Wings, results in neck, shoulder, and dorsal pain relief. The device indeed permits the discharge of the force developed from the weight lift from the UT to the LT, relaxing the former muscle. Given that shoulder posture has the ability to both facilitate and suppress specific motor pathways [14-16], the possibility that, during the proposed motor task, a facilitated pathway would, in principle, make the LT muscle fundamentally easier to activate than the UT, needs to be considered. Consequently, the appropriate selection of upper limb movement, combined with the Angel's Wings, may prove beneficial in rehabilitation environments to help selectively modulate the relative accessibility of specific muscles and thus retrain motor control.

It is important to consider that, in the tested population of employees, there were really different professionals, with different styles of life. A factor that is common for all categories of professionals, for physically demanding jobs and sedentary jobs, is the perception of pain in the portions of the body considered in this study. The pain frequently starts from the neck and then spreads to other parts of the body. Thus, for example, pilots and the pit stop laborer suffer from neck pain for different reasons. The neck of a pilot is frequently stressed for the types of accelerations and the forces to which they are subjected. Instead, the neck of a pit stop laborer is stressed for the type of movements they have to do during the changing of the tires in a Formula 1 race where time is a luxury they cannot afford. On the other hand, for example, the neck of a desk worker, i.e., a sedentary worker, is frequently stressed for an altered and repetitive posture assumed during the workday. In all of these circumstances, the use of the Angel's Wings appears to be a good method to counteract the pain and to decrease it in the cases where the pain is already present. 
Today, with the changing of lifestyles, many people, including several categories of workers, have "postural defects" characterized mainly by altered distribution of muscle mass. All of this becomes a tendency to assume a crouched, and in some cases painful, position forward, characterized by shoulders moved forward.

In subjects who perform motor tasks daily (morning and evening) for 30 days, via MRI images, we demonstrated a realignment of the cervico-dorsal column between the pre- and post-training phases due to a variation of only a few degrees in its curvatures. Moreover, an improvement in posture is shown because, additionally, the gibbus, caused by a kyphotic posture, appears blunt. This data is important, considering the fact that the training period was 30 days, a relatively short period of time.

In the VAS results, we can observe that, in general, in the sample of workers considered in this study (73 subjects), many people suffer from neck and shoulder pain, regardless of gender, age, level of training, or, of course, main occupation. In particular, in our experience, we found that most people in this study (60 in a group of 73) tend to suffer from neck pain in a moderate way-in many cases, radiating to the shoulders (49 in a group of 73). As reported in the graph in Figure 4, the pain also sometimes radiates in the dorsal area. However, as reported in the graphs of the VAS results, there was pain relief, in the considered areas, reported by the population participating in this study after only one session of training. We consider these results important, but we think there is a need of a period of training with a larger number of sessions for each subject to see if the training with this device can effectively stabilize the results of the pain relief in the neck, shoulders, and dorsal area.

The main limits of our study are that we do not have a randomized control trial, and, in relation to time, we can only conclude for a maximum period of 30 days. Another limitation is that we did not compare the effect of this exercise with other exercises (i.e., the pull-down exercise). Yet another limit of this study is that three MRI cases are not statistically significant and that the data needs to be verified in a larger sample of subjects. One more limit of this study is that our results presentation is not based on physiological evidence (i.e., blood circulation) and is discussed in light of the many limitations of the time frame for tissue adaptation, longevity of the outcome, and possible placebo effects.

\section{Conclusions}

In conclusion, our results demonstrate that the participants that used the "Angel's Wings" device showed lower neck pain and a postural correction of cervico-dorsal column structure. Moreover, the use of this device appears to be a really good compromise between the importance of obtained benefits and the time of use (only a few minutes per session). This finding provides some preliminary support for such an exercise in the treatment programs of neck pain patients. Further studies are needed to establish the effects of the use of Angel's Wings for a longer period of time.

Author Contributions: Luca Valerio Messa invented and patented the device Angel's Wings used in this study, designed the study and performed the experiments; Luca Valerio Messa and Federica Ginanneschi analyzed the data and wrote the paper; Alessandro Biffi, Fredrick Fernando and Alessandro Rossi supervised the study.

Conflicts of Interest: In this paper, there is a patented device used for the study. The authors knew this before starting the study and agreed to proceed with the study and the publication of the obtained results. The authors declare no conflict of interests relative to the presence in the paper of a patented device used for the study. The authors declare no other conflict of interests.

\section{References}

1. Australian Government Department of Health and Ageing. Australia's Physical Activity \& Sedentary Behaviour Guidelines for Adults (18-64 years) 2014. Available online: http:/ /www.health.gov.au/internet/ main/publishing.nsf/content/health-pubhlth-strateg-phys-act-guidelines (accessed on 15 September 2015).

2. Nindl, B.C.; Sharp, M.A. Physical Training for Improved Occupational Performance. American College of Sports Medicine. www.acsm.org. Available online: https://www.acsm.org/docs/currentcomments/rtforio.pdf (accessed on 15 September 2015). 
3. UK Government Department of Health. UK Physical Activity Guidelines: adults (19-64 years). In: Guidance from the Chief Medical Office (CMO) (ed.) July 2011. Available online: https:/ /www.gov.uk/government/ publications/uk-physical-activity-guidelines (accessed on 15 September 2015).

4. Hanvold, T.N.; Wærsted, M.; Mengshoel, A.M.; Bjertness, E.; Stigum, H.; Twisk, J.; Veiersted, K.B. The effect of work-related sustained trapezius muscle activity on the development of neck and shoulder pain among young adults. Scand. J. Work Environ. Health 2013, 39, 390-400. [CrossRef] [PubMed]

5. Seefeldt, V.; Malina, R.M.; Clark, M.A. Factors affecting levels of physical activity in adults. Sports Med. 2002, 32, 143-168. [CrossRef] [PubMed]

6. Buckle, P.W.; Devereux, J.J. The nature of work-related neck and upper limb musculoskeletal disorders. Appl. Ergon. 2002, 33, 207-217. [CrossRef]

7. Falla, D.L.; Jull, G.A.; Hodges, P.W. Patients with neck pain demonstrate reduced electromyographic activity of the deep cervical flexor muscles during performance of the craniocervical flexion test. Spine 2004, 29, 2108-2114. [CrossRef] [PubMed]

8. Thorn, S.; Søgaard, K.; Kallenberg, L.A.; Sandsjö, L.; Sjøgaard, G.; Hermens, H.J.; Kadefors, R.; Forsman, M. Trapezius muscle rest time during standardised computer work-A comparison of female computer users with and without self-reported neck/shoulder complaints. J. Electromyogr. Kinesiol. 2007, 17, 420-427. [CrossRef] [PubMed]

9. Bandy, W.D.; Sanders, B. Therapeutic Exercise: Techniques for Intervention; Lippincott Williams and Wilkins: Baltimore, MD, USA, 2001.

10. Messa, L.V.; Barberis, F.; Paradiso, C.; Paddeu, S.; Mardessich, S.; Arrigucci, U.; Bonifazi, M. Evidence of the Possibility to Contract the Lower Trapezius, Relaxing the Upper Trapezius, and Implications on Posture through the use of an Innovative Mechanical Device for Physical Training. In Proceedings of the ICSPORTS, Rome, Italy, 24-26 October 2014.

11. Cools, A.M.; Dewitte, V.; Lanszweert, F.; Notebaert, D.; Roets, A.; Soetens, B.; Cagnie, B.; Witvrouw, E.E. Rehabilitation of scapular muscle balance: Which exercises to prescribe? Am. J. Sports Med. 2007, 35, 1744-1751. [CrossRef] [PubMed]

12. Janda, V.L. Muscles and Motor Control in Cervicogenic Disorders: Assessment and Management. Physical Therapy of the Cervical and Thoracic Spine, 2nd ed; Churchill Livingstone: Philadelphia, PA, USA, 1994.

13. Haskell, W.L.; Lee, I.M.; Pate, R.R.; Powell, K.E.; Blair, S.N.; Franklin, B.A.; Macera, C.A.; Heath, G.W.; Thompson, P.D.; Bauman, A. American Heart AssociationPhysical activity and public health: Updated recommendation for adults from the American College of Sports Medicine and the American Heart Association. Circulation 2007, 116, 1081-1093. [PubMed]

14. Ginanneschi, F.; Del Santo, F.; Dominici, F.; Gelli, F.; Mazzocchio, R.; Rossi, A. Changes in corticomotor excitability of hand muscles in relation to static shoulder positions. Exp. Brain Res. 2005, 161, 374-382. [CrossRef] [PubMed]

15. Ginanneschi, F.; Dominici, F.; Biasella, A.; Gelli, F.; Rossi, A. Changes in corticomotor excitability of forearm muscles in relation to static shoulder positions. Brain Res. 2006, 1073-1074, 332-338. [CrossRef] [PubMed]

16. Mogk, J.P.; Rogers, L.M.; Murray, W.M.; Perreault, E.J.; Stinear, J.W. Corticomotor excitability of arm muscles modulates according to static position and orientation of the upper limb. Clin. Neurophysiol. 2014, 125, 2046-2054. [CrossRef] [PubMed]

(C) 2016 by the authors; licensee MDPI, Basel, Switzerland. This article is an open access article distributed under the terms and conditions of the Creative Commons by Attribution (CC-BY) license (http://creativecommons.org/licenses/by/4.0/). 